\title{
Crecimiento, producción y calidad de fruta en feijoa [Acca sellowiana (0.Berg) Burret] en respuesta al nitrato de potasio, fosfato de potasio y ethephon
}

\section{Pineapple guava [Acca sellowiana (0.Berg) Burret] growth, production and fruit quality in response to potassium nitrate, potassium phosphate and ethephon}

LUIS FERNANDO COMBARIZA'

CÉSAR AUGUSTO NEIRA ${ }^{1}$

GERHARD FISCHER ${ }^{2}$

GUILLERMO CORREDOR ${ }^{3}$

ÓMAR CAMILO QUINTERO 4

Árbol de feijoa en estadio
de botón floral y floración.
Foto: G. Fischer

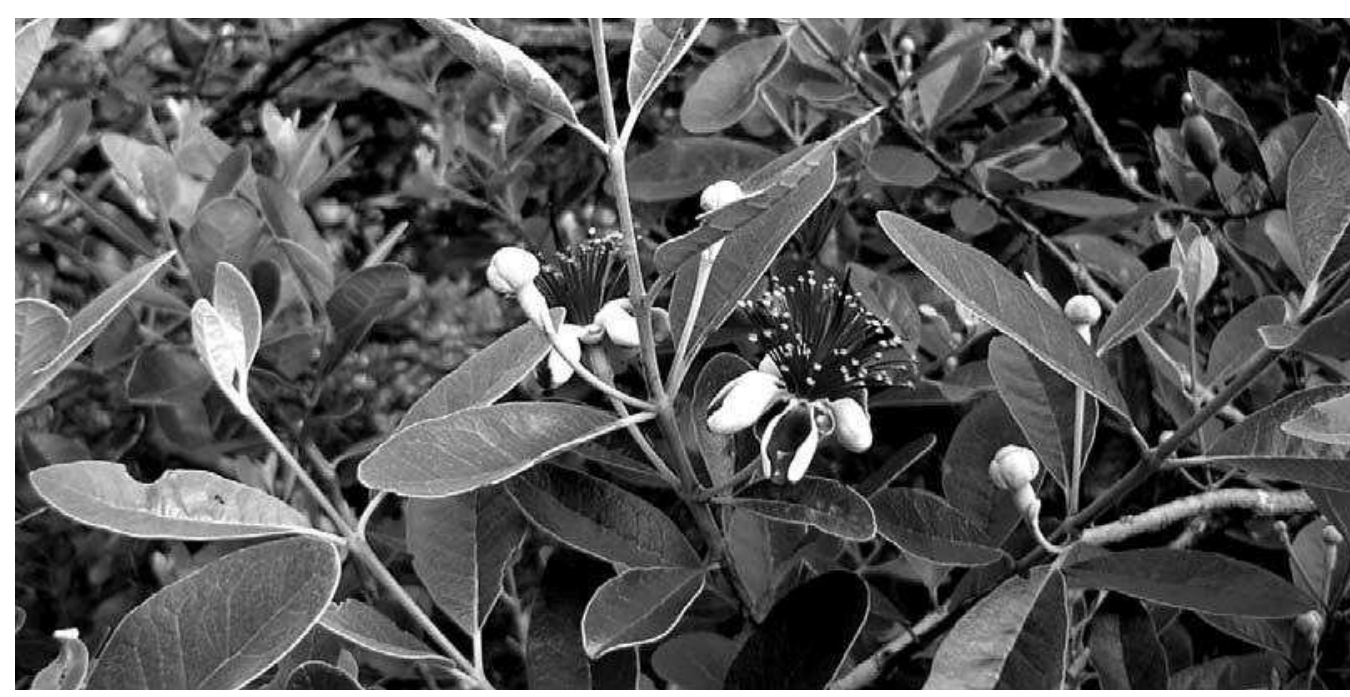

\section{RESUMEN}

En La Vega (Cundinamarca, Colombia; $2.350 \mathrm{msnm}$, temperatura, humedad relativa y precipitación anual promedio de $17^{\circ} \mathrm{C}, 85 \%$ y $1.423 \mathrm{~mm}$, respectivamente), se estudió el efecto de tres productos químicos, usados en la inducción floral, en árboles de feijoa ('Clon 41' o 'Ouimba'), los cuales tenían 6 años de edad. Los productos aplicados fueron los fertilizantes foliares $\mathrm{KNO}_{3}$ (nitrato de potasio 13-0-46 ${ }^{\circledR}$ en concentraciones de 1,0\%, 1,5\%, 2,0\%), $\mathrm{KH}_{2} \mathrm{PO}_{4}$ (fosfato de potasio; Cosmofoliar 0-32-43 ${ }^{\circledR} ; 0,5 \%, 0,75 \%, 1,0 \%$ ) y ethephon (Ethrel ${ }^{\circledR} 48$ SL; 100, 150, 200 $\mathrm{mg} \cdot \mathrm{L}^{-1}$ ) cuyos efectos fueron evaluados durante el desarrollo, hasta la madurez fisiológica del fruto, mediante la determinación de la producción y la calidad de este. En relación con el tamaño longitudinal y transversal del fruto, el $\mathrm{KH}_{2} \mathrm{PO}_{4}$, al 0,5\%, seguido por el tratamiento ethephon, a $100 \mathrm{mg} \cdot \mathrm{L}^{-1}$, presentó la mejor respuesta en comparación con los otros tratamientos, con una producción óptima de frutos calidad exportable. Así mismo, 0,5\% $\mathrm{KH}_{2} \mathrm{PO}_{4}$ produjo el mayor número de frutos cuajados y cosechados, mientras el ethephon, al $100 \mathrm{mg} \cdot \mathrm{L}^{-1}$ tendió a originar los frutos de mayor peso fresco. Los productos aplicados no presentaron una alteración en las variables químicas (pH, sólidos solubles totales y acidez total titulable) de los frutos cosechados.

Ingenieros Agrónomos, Facultad de Agronomía, Universidad Nacional de Colombia, Bogotá (Colombia).

lfcombarizar@unal.edu.co; caneirac@unal.edu.co

2 Profesor Asociado, Facultad de Agronomía, Universidad Nacional de Colombia, Bogotá (Colombia).

gfischer@unal.edu.co

3 Profesor Asociado (r), Facultad de Agronomía, Universidad Nacional de Colombia, Bogotá (Colombia).

guiart7co@yahoo.es

4 Biólogo, DisFruta las Feijoas, Bogotá (Colombia).overfeijoas@gmail.com 


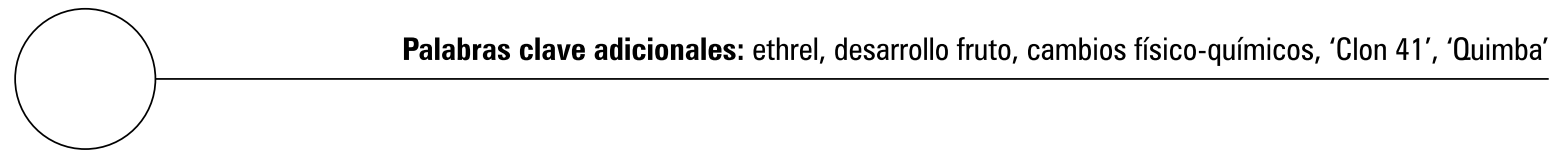

\section{ABSTRACT}

In La Vega (Cundinamarca, Colombia; $2,350 \mathrm{~m}$ above sea level, $17^{\circ} \mathrm{C}$ average temperature, $85 \%$ relative humidity, and $1,423 \mathrm{~mm}$ annual precipitation), the effect of three chemical products, used for floral induction, on 6 yearold pineapple guava trees ('Clone $41^{\prime}$ ' or 'Quimba') were studied. The applied chemical agents were the foliar fertilizers $\mathrm{KNO}_{3}$ (potassium nitrate $13-0-46^{\circledR} 1 \%, 1.5 \%, 2 \%$ ), $\mathrm{KH}_{2} \mathrm{PO}_{4}$ (potassium phosphate; Cosmofoliar 0-32$43^{\circledR} ; 0.5 \%, 0.75 \%, 1.0 \%$ ), and ethephon (Ethrel ${ }^{\circledR} 48 \mathrm{SL} ; 100,150,200 \mathrm{mg} \cdot \mathrm{L}^{-1}$ ) their effects were evaluated during fruit development, until physiological maturity, determining fruit production and quality. Regarding the longitudinal and cross-sectional size of the fruit, $\mathrm{KH}_{2} \mathrm{PO}_{4}$ at $0.5 \%$, followed by the $100 \mathrm{mg} \cdot \mathrm{L}^{-1}$ ethephon treatment, responded better compared to the other treatments, with an optimal production of fruits of exportable quality. Similarly, $0.5 \% \mathrm{KH}_{2} \mathrm{PO}_{4}$ produced the highest number of set and harvested fruits. Ethephon at $100 \mathrm{mg} \cdot \mathrm{L}^{-}$ ${ }^{1}$ tended to originate the greatest fresh weight fruits. The applied products did not present any alteration in their chemical variables ( $\mathrm{pH}$, total soluble solids and total titratable acidity) of harvested fruits.

Additional key words: ethrel, fruit development, physicochemical changes, 'Clone 41', 'Quimba'.

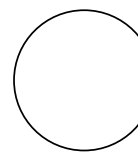

INTRODUCCIÓN

La importancia de la feijoa [Acca sellowiana (O.Berg) Burret, Mirtaceae] radica en su buena adaptación a las zonas tropicales y en especial a la región andina colombiana. Es una planta de origen subtropical, cuya fruta por varios años ha tenido excelente aceptación en los mercados nacionales e internacionales, bien sea para consumo en fresco o para agroindustria, por lo cual, se ha convertido en necesidad para el agricultor, contar con una producción constante para lograr un buen abastecimiento al mercado, más aún a nivel de exportación. La planta se caracteriza por su alta adaptabilidad a zonas climáticas, y en cultivos comerciales en Colombia se le encuentra en altitudes entre 1.800 y $2.650 \mathrm{msnm}$, temperatura entre 13 y $21^{\circ} \mathrm{C}$ (con un promedio óptimo de $16^{\circ} \mathrm{C}$ ), precipitación anual de 700 a $1.200 \mathrm{~mm}$, $1.500 \mathrm{~h}$ brillo solar/año y humedad relativa promedio alrededor de 70\% (Fischer, 2003).
En Colombia se ha aumentado el área de cultivo de feijoa en los últimos 10 años como una alternativa viable para la diversificación de la producción agrícola (Corporación Colombia Internacional, 2000), mientras los reportes que se tienen en investigaciones acerca de los cambios fisiológicos en esta especie son muy pocos. Los resultados de las primeras investigaciones en Colombia, realizadas por Quintero (2003), definieron que los cultivares de mayor producción en el departamento de Cundinamarca se encuentran en la finca CENAF (Centro Nacional de la Feijoa) y son el 'Clon 41' (Quimba), 'CENAF 8-4' y 'CENAF 15-1'. El cultivo de la feijoa tiene una ventaja comparativa alta para Colombia, el cual, por ser un país tropical, podría llegar a garantizar un suministro constante durante todo el año, a pesar de lo cual, la producción ocurre en períodos definidos, lo que genera sobreoferta en épocas de 
cosecha y escasez en algunos períodos del año, y de esta manera desbalances en la cadena productiva (Valderrama et al., 2005).

El tamaño y calidad del fruto dependen de los suministros adecuados y regulares de agua. En períodos secos prolongados, la planta reacciona con caída de hojas, flores y frutos. En feijoa, los mejores resultados en floración, polinización y llenado del fruto se presentan con libre exposición a la radiación solar, siempre y cuando no existan condiciones secas y temperaturas altas (Fischer, 2003).

Las flores de muchos cultivares son autofértiles, permitiendo la polinización cruzada, aumentando el porcentaje de los frutos cuajados, razón por la cual, se deben sembrar diferentes cultivares o clones en la misma plantación (Quintero, 2003). La caída de flores y frutos, según Schumann y Lüdders (1992), es frecuente en los casos de una deficiente polinización por pájaros y por la protoginia (maduración del estigma antes de las anteras).

El fruto es una baya de forma variable, prevaleciendo la ovoidal, con pesos hasta más de $100 \mathrm{~g}$, epidermis de color brillante o verde grisáceo, aun en su estado de madurez fisiológica (Pachón y Quintero, 1992). De acuerdo a lo encontrado por Galvis (2002), los frutos, normalmente, muestran una curva de crecimiento (en tamaño o peso) de forma sigmoidal simple, pero esta según el cultivar y las condiciones agroecológicas puede ser también sigmoidal doble. El desarrollo del fruto dura de 120 a 150 días, presentándose durante los primeros 40 días un desarrollo lento, terminando la maduración con un climaterio. La feijoa presenta una madurez óptima de cosecha inmediatamente antes de su caída natural (Seymor et al., 1993).

En condiciones agroecológicas favorables, con un manejo de huerto racional y con la selección de cultivares fértiles, la planta puede llegar a producir durante 30 o más años. La feijoa inicia su etapa productiva a los tres años, si se ha hecho la propagación por medios vegetativos (Fischer, 2003).

Una de las aplicaciones más atractivas de la producción forzada es obtener la cosecha fuera de la época normal de producción para comercializarla a mejor precio. También es posible extender la oferta del producto en el mercado durante más tiempo, además de aumentar el rendimiento anual por unidad de superficie. Sin embargo, la base genética, los procesos fisiológicos y la manera en que estos son afectados por este tipo de tratamientos no han sido comprendidos completamente (Weigel, 1995).

El potasio fuerza la formación de fosfatos energéticos que al tiempo son necesarios para la formación floral (Feucht, 1982). El número alto de botones florales y una abundante fertilidad están ligados a un óptimo suministro con fosfato. La deficiencia de este elemento origina floración escasa (Agustí, 2003).

En la localidad de Tena (Cundinamarca), la aplicación del $\mathrm{KNO}_{3}$, ethephon o la poda no registraron diferencias significativas en cuanto a la inducción floral de la pitahaya amarilla (Hylocereus triangularis), sin embargo, se notó mejor respuesta en la calidad de frutos en plantas tratadas con $\mathrm{KH}_{2} \mathrm{PO}_{4}$ (Colorado y Manjarres, 1992). En feijoa, García et al. (2008) encontraron que el $\mathrm{KH}_{2} \mathrm{PO}_{4}$ al $0,5 \%$ produjo el mayor número de flores formadas.

Algunas experiencias de inducción floral han intentado controlar la fructificación en mango (Mangifera indica L.), mostrando que ethephon (200 mg $\cdot \mathrm{L}^{-1}$ ) puede ser usado para inducir la floración y la fructificación durante los años "off" (Chacko y Randhawa, 1974). En guayaba, la doble aspersión con Ethrel $\left(1 \mathrm{~mL} \cdot \mathrm{L}^{-1}\right)$ resultó en un mayor número de flores y frutos por planta comparado con aplicaciones de nitrato de amonio, micronutrientes y estrés hídrico (Castelán-Estrada y Becerril-Román, 1994). 
Las aplicaciones de nutrientes vía foliar en estados fenológicos claves del cultivo define Lovatt (1999) en 5 a 30 veces más eficientes que las aplicaciones edáficas, teniendo en cuenta que las épocas de floración y cuajamiento del fruto tienen una alta demanda nutricional.

El presente trabajo tuvo como objetivo evaluar la respuesta de la feijoa a la aplicación de los fertilizantes foliares $\mathrm{KNO}_{3}$ y $\mathrm{KH}_{2} \mathrm{PO}_{4}$ y la fitohormona ethephon, a tres dosis cada uno, desde antesis hasta madurez fisiológica del fruto, estudiando su crecimiento, desarrollo, calidad y producción.

\section{MATERIALES Y MÉTODOS}

La realización del estudio tuvo lugar en el municipio de la Vega (Cundinamarca, Colombia), vereda El Chuscal, finca El Cortijo, situada a 04³9' latitud norte y $71^{\circ} 57^{\prime}$ longitud oeste, ubicada a $2.350 \mathrm{msnm}$, con temperatura de $17^{\circ} \mathrm{C}$, humedad relativa de $85 \%$ y precipitación anual promedio de $1.423 \mathrm{~mm}$, respectivamente, y con una cantidad anual de horas brillo solar de 1.377 (IDEAM, 2002). Según clasificación Holdridge (1996) esta zona pertenece al bosque húmedo montano bajo (bh-MB) (IGAC, 2.000). Se eligió el lote número 5 $\left(9.750 \mathrm{~m}^{2}\right)$ de la finca, el cual presenta una pendiente de $25 \%$ con 975 plantas, sembradas a una distancia de 4 × 2,5 m. La toma de datos en campo tuvo una duración de 5 meses empezando en los últimos días del mes de junio y culminando en el mes de noviembre de 2006.

Las plantas tratadas fueron del cultivar Clon 41 (Quimba) que al inicio del experimento tenían una edad de 6 años y sus ramas presentaban agobio forzado. La selección de los árboles se realizó de forma aleatoria, utilizando tres ramas del tercio inferior del árbol, a una altura promedio con respecto al suelo de $60 \mathrm{~cm}$. Luego de la selección se realizó la labor de la poda de las cuatro ramas del primer piso para estimular la formación de nuevos botones florales (Quintero, 1993). Los árboles seleccionados se encontraban en la fase reproductiva en la que si la poda se realizara (teniendo el árbol botones florales, flores, cuajas, frutos o todos a la vez), la respuesta sería la emisión de ramas nuevas con más botones florales (Quintero, 2003).

Se utilizó un diseño de bloques completos al azar con 10 tratamientos y tres repeticiones debido a que el terreno presentaba una pendiente considerable y los tratamientos fueron distribuidos al azar en cada uno de los bloques; la unidad experimental estuvo constituida por un árbol. Los tratamientos fueron: (1) Nitrato de potasio 13-0$46^{\circledR}\left(\mathrm{KNO}_{3}\right) 1 \%$; (2) $\mathrm{KNO}_{3} 1,5 \%$; (3) $\mathrm{KNO}_{3} 2 \%$; (4) Cosmofoliar 0-32-43 ${ }^{\circledR}\left(\mathrm{KH}_{2} \mathrm{PO}_{4}\right)$ 0,5\%; (5) $\mathrm{KH}_{2} \mathrm{PO}_{4}$ $0,75 \%$; (6) $\mathrm{KH}_{2} \mathrm{PO}_{4} 1 \%$; (7) Ethrel $^{\circledR} 48 \mathrm{SL}$ (Ethephon) $100 \mathrm{mg} \cdot \mathrm{L}^{-1}$; (8) Ethephon $150 \mathrm{mg} \cdot \mathrm{L}^{-1}$ (9) Ethephon $200 \mathrm{mg} \cdot \mathrm{L}^{-1}$ y (10) Testigo (sin aplicación).

Para una mayor eficacia de los productos inductores se empleó el coadyuvante COSMO-IND ${ }^{\circledR}(1$ $\left.\mathrm{cm}^{3} \cdot \mathrm{L}^{-1}\right)$. Se dejó un árbol intermedio entre cada tratamiento (árbol borde), para evitar la deriva de la aplicación de los tratamientos ya que puede influir o enmascarar la respuesta del árbol al tratamiento aplicado.

Al momento de realizar la poda se aplicaron los productos en forma asperjada al tercio inferior del árbol, mediante una bomba manual (1,6 L/ árbol). Una vez aplicados los tratamientos, se repitieron cada 15 días durante los dos meses seguidos. Las aplicaciones se realizaron en las horas de la mañana, cuando la temperatura no era muy elevada. Las labores de manejo (fertilización edáfica, deshierba y control fitosanitario) se hicieron de forma similar para todo el lote de la finca. Los muestreos se llevaron a cabo cada ocho días a partir del cuajamiento de los frutos hasta el final del ensayo.

En campo, se usó un diseño en bloques completos al azar, con 10 tratamientos y tres repeticiones (tabla 1). La unidad experimental estuvo constituida por un árbol del cual se escogieron tres ramas del tercio inferior y cada rama con tres 
frutos distribuidos en la misma. Posteriormente con los datos obtenidos se realizó un análisis estadístico, que incluyó análisis de varianza (ANAVA). Se efectuaron comparaciones entre grupos de tratamientos a través de contrastes, pruebas Tukey y análisis de regresión; para establecer el mejor tratamiento, se halló el coeficiente de determinación y la respectiva ecuación.

El análisis de calidad del fruto se llevó a cabo en el Laboratorio de Fisiología de Cultivos de la Facultad de Agronomía de la Universidad Nacional de Colombia, Bogotá, evaluando (a) diámetro transversal y (b) diámetro longitudinal del fruto (desde cuajado hasta la cosecha, (c) peso fresco del fruto cosechado, (d) número total de frutos cosechados, (e) sólidos solubles totales (SST) del fruto cosechado, (f) $\mathrm{pH}$ del fruto cosechado y ( $\mathrm{g}$ ) acidez total titulable (ATT) del fruto cosechado.

Posterior a la aplicación de los productos químicos se procedió a la toma de datos, la cual se realizó por un período de 154 días, según lo reportado por Fischer (2003) y ratificado por Rodríguez et al. (2006a), estimando un tiempo de madurez fisiológica de cerca de 156 días. Los datos se tomaron con intervalos de 15 días. Los diámetros transversal y longitudinal de los frutos se obtuvieron con un calibrador Vernier (Maub, de 0,05 $\mathrm{mm}$ de precisión), el peso fresco mediante una balanza electrónica Mettler P1200 (precisión 0,01 g). Los sólidos solubles totales (grados Brix) se determinó con un refractómetro Carl Zeiss Jena con escala de 0 a $100^{\circ} \mathrm{Brix}$, el valor de $\mathrm{pH}$ con un potenciómetro Orión, modelo 420. La acidez total titulable, mediante titulación con $\mathrm{NaOH} 0,1 \mathrm{~N}$ hasta pH 8,2, la cual se expresó como porcentaje de ácido cítrico (Rodríguez et al., 2006b). Para la determinación de las variables en laboratorio se tomaron tres frutos por tratamiento.

El presente estudio fue una continuación del experimento de García et al. (2008) quienes evaluaron el efecto de estos mismos productos químicos en la inducción floral y el cuajado del fruto de la feijoa en los mismos árboles.

\section{RESULTADOS Y DISCUSIÓN}

\section{Diámetro transversal del fruto}

De acuerdo con el análisis de varianza (ANAVA) se encontraron diferencias significativas entre los tratamientos para esta variable, determinando que el mejor fue el $\mathrm{KH}_{2} \mathrm{PO}_{4}$ al 0,5\%. Esto se demuestra estadísticamente por medio de comparaciones múltiples que dejan ver una tendencia a aumentar el diámetro transversal del fruto con el tratamiento $\mathrm{KH}_{2} \mathrm{PO}_{4}$ al 0,5\% con respecto a los otros tratamientos. Adicional a esto en la curva de crecimiento del fruto de feijoa, expresada como diámetro transversal en un modelo lineal (figura 1), se observa una mejor respuesta en este tratamiento al final del ensayo (tabla 1). Los coeficientes de determinación $\left(R^{2}\right)$ para el diámetro transversal de los frutos fueron altos $(>86)$, lo cual se atribuye al transcurso del tiempo.

En la tabla 1 se observa que el tratamiento $\mathrm{KH}_{2} \mathrm{PO}_{4}$ al 0,5\%, fue estadísticamente diferente a la aplicación de $\mathrm{KNO}_{3}$ al 1,0\%, $\mathrm{KH}_{2} \mathrm{PO}_{4}$ al 1,0\%, y ethephon a $200 \mathrm{mg} \cdot \mathrm{L}^{-1}$. La figura 1 muestra que los frutos tienen un crecimiento y desarrollo acorde con lo indicado por Galvis (2003) y Rodríguez et al. (2006a), quienes reportaron que el diámetro del fruto de feijoa consta de tres fases, la primera de un crecimiento lento hasta los 70 días, la segunda etapa tiene un período de mayor crecimiento, desde los 70 días hasta los 126 días y la tercera etapa tiene un crecimiento rápido, y va desde los 126 días hasta la madurez fisiológica (aproximadamente 156 días) lo cual concuerda con lo encontrado en el presente estudio.

En las primeras etapas del crecimiento no se aprecia aumento significativo en el diámetro transversal (figura 1), lo cual concuerda con lo reportado por Salisbury y Ross (2003) y Grierson (2002). Es para tener en cuenta que el potasio que forma parte del $\mathrm{KH}_{2} \mathrm{PO}_{4}$ fue complementario a la acción favorable del fósforo, asociando el potasio como un elemento importante para la producción de frutos de mayor tamaño (Yahía et 
Tabla 1. Efecto del nitrato del potasio $\left(\mathrm{KNO}_{3}\right)$, el fosfato de potasio $\left(\mathrm{KH}_{2} \mathrm{PO}_{4}\right)$ y ethephon (Ethrel) sobre el díametro transversal del fruto de feijoa, 'Clon 41' (Quimba).

\begin{tabular}{|l|c|c|c|}
\multicolumn{1}{|c|}{ Tratamiento } & Dosis & $\begin{array}{c}\text { Diámetro transversal } \\
(\mathrm{mm})\end{array}$ & $\begin{array}{c}\text { Diámetro longitudinal } \\
(\mathrm{mm})\end{array}$ \\
\hline $\mathrm{KNO}_{3}$ & $1,0 \%$ & $30,4 \mathrm{bcd}$ & $44,2 \mathrm{ab}$ \\
\hline $\mathrm{KNO}_{3}$ & $1,5 \%$ & $38,8 \mathrm{abc}$ & $61,5 \mathrm{ab}$ \\
\hline $\mathrm{KNO}_{3}$ & $2,0 \%$ & $32,9 \mathrm{abcd}$ & $49,0 \mathrm{ab}$ \\
\hline $\mathrm{KH}_{2} \mathrm{PO}_{4}$ & $0,5 \%$ & $44,9 \mathrm{a}$ & $66,0 \mathrm{a}$ \\
\hline $\mathrm{KH}_{2} \mathrm{PO}_{4}$ & $0,75 \%$ & $38,9 \mathrm{abc}$ & $57,0 \mathrm{ab}$ \\
\hline $\mathrm{KH}_{2} \mathrm{PO}$ & $1,0 \%$ & $25,1 \mathrm{~d}$ & $39,3 \mathrm{~b}$ \\
\hline Ethephon & $100 \mathrm{mg} \cdot \mathrm{L}^{-1}$ & $39,4 \mathrm{abc}$ & $62,1 \mathrm{ab}$ \\
\hline Ethephon & $150 \mathrm{mg} \cdot \mathrm{L}^{-1}$ & $34,0 \mathrm{abcd}$ & $56,0 \mathrm{ab}$ \\
\hline Ethephon & $200 \mathrm{mg} \cdot \mathrm{L}^{-1}$ & $27,1 \mathrm{~cd}$ & $51,4 \mathrm{ab}$ \\
\hline Testigo & 0 & $42,8 \mathrm{ab}$ & $64,5 \mathrm{ab}$ \\
\hline
\end{tabular}

*Promedios con letras distintas son significativamente diferentes, según la prueba de Tukey $(P \leq 0,05)$.

al., 2006), como confirma la concentración de $\mathrm{KH}_{2} \mathrm{PO}_{4}$ al $5 \%$ que fue importante para el aumento del diámetro transversal (figura 1). El fósforo en la concentración del $5 \%$ del fosfato de potasio encontró su óptima concentración, teniendo en cuenta que el nivel de este elemento puede ser todavía más elevado al comienzo del ciclo del crecimiento del fruto, cuando una alta división celular está ocurriendo en hojas desarrollándose y en frutos pequeños (Neilsen y Neilsen, 2003).

Salisbury y Ross (2003) afirman que en plantas, donde el fosfato se encuentra en abundancia, se acelera el proceso de maduración, y se retarda cuando hay escasez de este elemento. El fosfato se redistribuye con facilidad en la mayor parte

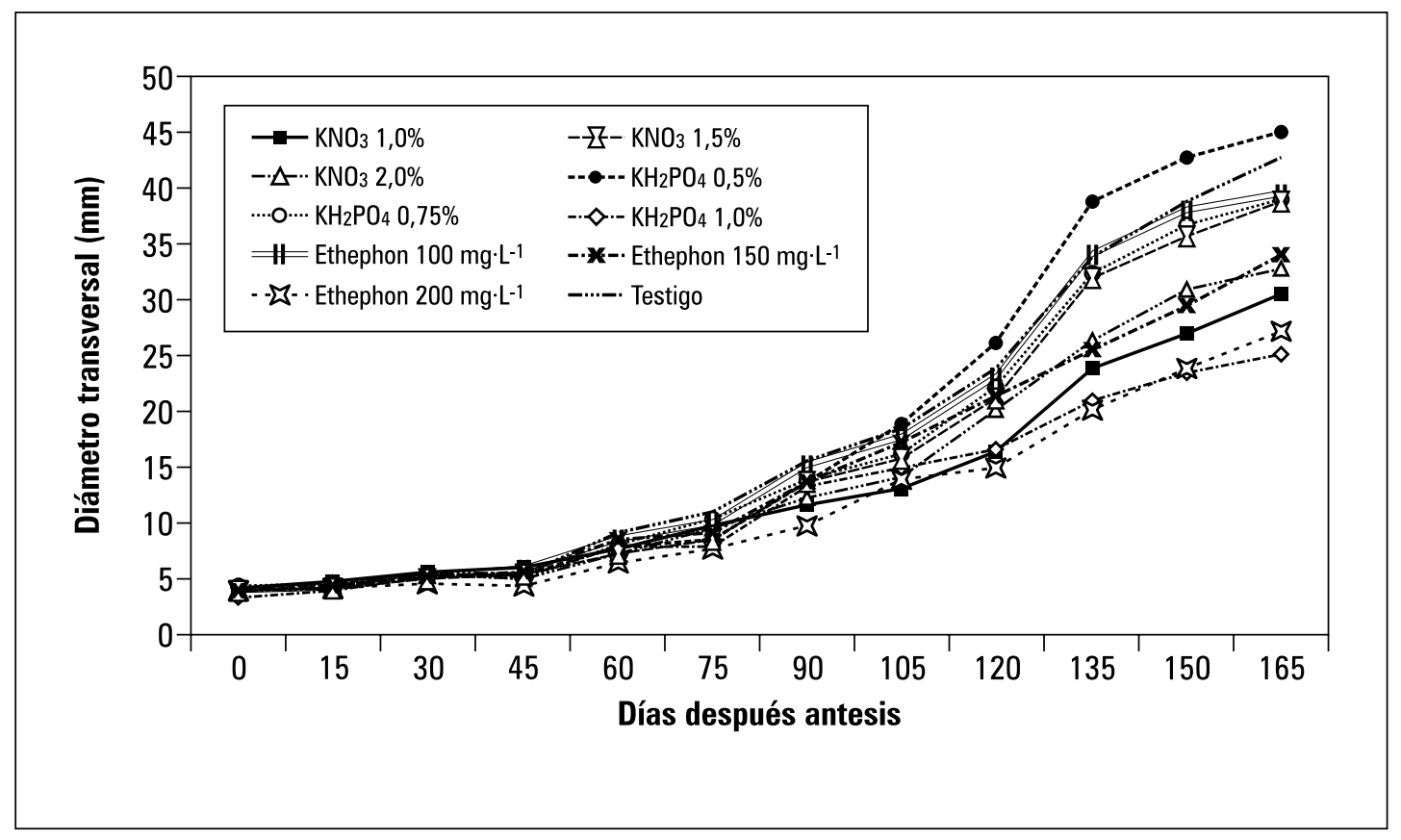

Figura 1. Efecto del nitrato de potasio $\left(\mathrm{KNO}_{3}\right)$, fosfato de potasio $\left(\mathrm{KH}_{2} \mathrm{PO}_{4}\right)$ y ethephon (Ethrel) en el diámetro transversal del fruto de feijoa, 'Clon 41' (Quimba). 
de las plantas de un órgano a otro y se pierde en las hojas antiguas, acumulándose en hojas jóvenes, flores y semillas en desarrollo; además el fósforo es parte esencial de muchos glucofosfatos que participan en la fotosíntesis, la respiración y otros procesos metabólicos.

\section{Diámetro longitudinal del fruto}

De acuerdo con la interpretación del análisis de varianza se encontraron diferencias significativas entre los tratamientos para esta variable, determinando que el mejor de ellos fue el tratamiento $\mathrm{KH}_{2} \mathrm{PO}_{4}$ al 0,5\%, al igual que para la variable anterior. Esto se demuestra estadísticamente, donde las comparaciones múltiples dejan ver una diferencia significativa de este tratamiento con respecto a $\mathrm{KH}_{2} \mathrm{PO}_{4}$ al $1,0 \%$ y un comportamiento con tendencia superior a los demás tratamientos. Adicional a esto, en la curva de crecimiento longitudinal del fruto de feijoa (figura 2), se observa una mejor respuesta en este tratamiento al final del ensayo. Los coeficientes de determinación para el diámetro longitudinal fueron altos, lo cual se atribuye al transcurso del tiempo.
Como se observa en la figura 2 la regresión de cada tratamiento es lineal, donde, en las primeras etapas del crecimiento, no hubo aumento significativo en el diámetro longitudinal (Grierson, 2002). La causa aparente del incremento en el crecimiento a razón de $\mathrm{KH}_{2} \mathrm{PO}_{4}$ al 0,5\%, comparado con los demás tratamientos, puede deberse a que el fósforo es esencial en el metabolismo energético por su presencia en moléculas como el ATP, ADP, AMP y pirofosfato (Salisbury y Ross, 2003). El potasio, por su parte, es un activador de muchas enzimas que son esenciales en la fotosíntesis y la respiración, además de que activa enzimas necesarias para formar almidón y proteínas (Bhandal et al., 1988).

Por otra parte, excesos de fósforo, conducirán a un desarrollo anormal y reducido de las hojas, creando un desequilibrio por reducción de los órganos fuente para el llenado de frutos, además puede interferir en la asimilación de otros nutrientes como el zinc (Salisbury y Ross, 2003). De acuerdo con lo anterior, es posible que con aplicaciones menores o mayores de $\mathrm{KH}_{2} \mathrm{PO}_{4}$ al $0,5 \%$ no se obtenga un buen crecimiento del fruto

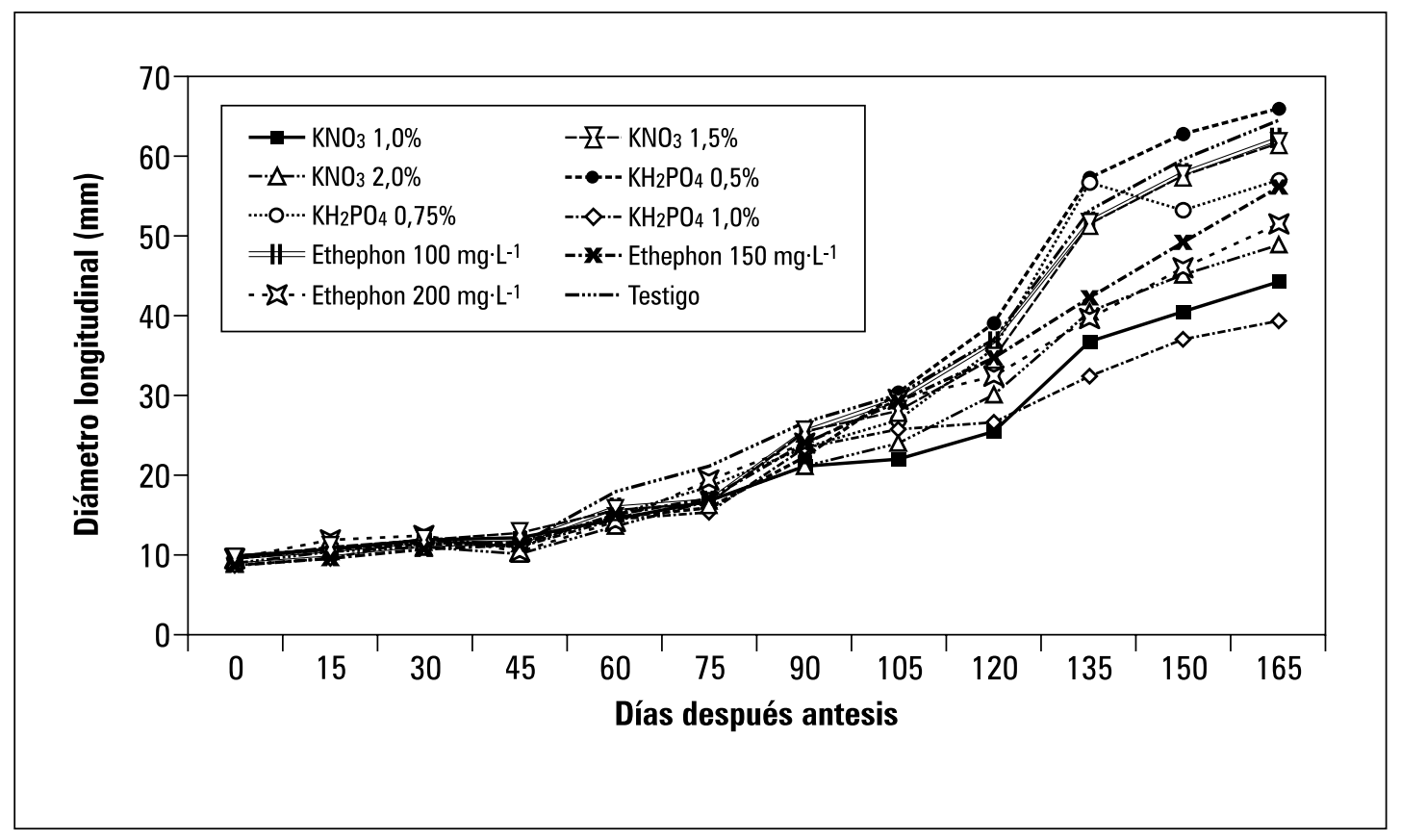

Figura 2. Efecto del nitrato de potasio $\left(\mathrm{KNO}_{3}\right)$, fosfato de potasio $\left(\mathrm{KH}_{2} \mathrm{PO}_{4}\right)$ y ethephon (Ethrel) en el diámetro longitudinal del fruto de feijoa, 'Clon 41' (Quimba). 
y se incurra en una deficiencia o exceso de este elemento en la planta, conllevando a una reducción en la producción y calidad del fruto.

\section{Peso fresco del fruto}

El tratamiento con mejor respuesta para el peso fresco del fruto, fue ethephon a $100 \mathrm{mg} \cdot \mathrm{L}^{-1}$ (tabla 2), sin embargo, no se encontró una diferencia significativa (Tukey, $P \leq 0,05$ ), presentándose, en comparación con las diferentes concentraciones del mismo producto, una alta diferencia entre la menor y la mayor dosis. En esta variable, el $\mathrm{KH}_{2} \mathrm{PO}_{4}$ al $5 \%$ no pudo arrojar mejores resultados $(72,0 \mathrm{~g})$ debido a que en este tratamiento el número de frutos fue el más alto (73 en las tres ramas evaluadas; tabla 4) por lo que se produjo un efecto de competencia entre ellos (Agustí, 2003).

Debido a que no existe una norma de calidad para la feijoa, se había puesto en marcha en el país por algunos agricultores y comercializadores el fijar una clasificación del fruto con base en el peso fresco, obteniendo fruta selecta $(>80 \mathrm{~g})$ con características tipo exportación y corriente (60-80 g), lo que concuerda con la mayoría de los tratamientos, excepto $\mathrm{KH}_{2} \mathrm{PO}_{4}$ al 0,75 y $1 \%$ y ethephon al $200 \mathrm{mg} \cdot \mathrm{L}^{-1}$.

\section{$\mathrm{pH}$ del fruto}

De acuerdo al análisis de varianza, en esta variable no hubo diferencia significativa de ningún tratamiento (tabla 2). Debido a que el pH es una característica fundamental para la calidad del fruto, los datos obtenidos en laboratorio oscilaron entre 2,83 y 3,08 lo cual concuerda con lo reportado por Galvis (2003), es decir que este fruto en procesos de transformación industrial no presentará ningún tipo de inconveniente. También, estos valores coinciden con los obtenidos por Rodríguez et al. (2006b), quienes reportaron que el clon Quimba conserva por mayor tiempo sus características organolépticas, con una mejor relación ácido-dulce, lo que concuerda con la calificación de sabor excelente para este clon ( $\mathrm{Ra}$ mírez, 1997).

\section{Sólidos solubles totales (SST) del fruto}

El análisis de varianza no mostró diferencia significativa entre los tratamientos, sin embargo observando que el tratamiento ethephon a 100 $\mathrm{mg} \cdot \mathrm{L}^{-1}$ tiende a los valores más altos (tabla 2 ).

Esta tendencia puede ser a causa del efecto del etileno, que acelera la maduración del fruto lo cual podría haber causado un aumento en los SST (Salisbury y Ross, 2003). La feijoa en estado verde presenta altas concentraciones de almidón el cual se hidroliza durante la maduración aumentando los niveles de los SST (Galvis, 2003).

\section{Acidez total titulable (ATT) del fruto}

De acuerdo al análisis de varianza no se observó diferencia significativa de los tratamientos (tabla 2). Según análisis de correlaciones, realizado por Rodríguez et al. (2006b) en feijoa, la ATT está inversamente correlacionada con los niveles de

Tabla 2. Efecto del nitrato de potasio $\left(\mathrm{KNO}_{3}\right)$, fosfato de potasio $\left(\mathrm{KH}_{2} \mathrm{PO}_{4}\right)$ y ethephon (Ethrel) en el peso fresco, el pH, los sólidos solubles totales (SST) y la acidez total (titulable ATT) del fruto de feijoa, 'Clon 41' (Quimba).

\begin{tabular}{|c|c|c|c|c|c|c|c|c|c|c|}
\hline \multirow{2}{*}{$\begin{array}{l}\text { Característica } \\
\text { fruto }\end{array}$} & \multicolumn{3}{|c|}{$\mathrm{KNO}_{3}(\%)$} & \multicolumn{3}{|c|}{$\mathrm{KH}_{2} \mathrm{PO}_{4}(\%)$} & \multicolumn{3}{|c|}{ Ethephon $\left(\mathrm{mg} \cdot \mathrm{L}^{-1}\right)$} & \multirow{2}{*}{ Testigo } \\
\hline & 1,0 & 1,5 & 2,0 & 0,5 & 0,75 & 1,0 & 100 & 150 & 200 & \\
\hline Peso fresco $(\mathrm{g})$ & $70,7 a b^{*}$ & $80,3 \mathrm{ab}$ & $62,9 \mathrm{ab}$ & $72,0 \mathrm{ab}$ & $45,7 \mathrm{ab}$ & $56,8 a b$ & $91,4 \mathrm{a}$ & $62,6 \mathrm{ab}$ & $56,3 \mathrm{ab}$ & $76,5 \mathrm{ab}$ \\
\hline $\mathrm{pH}$ & $3,01 \mathrm{a}$ & $3,08 \mathrm{a}$ & $2,09 \mathrm{a}$ & $2,89 a$ & $2,85 a$ & $2,86 \mathrm{a}$ & $2,83 \mathrm{a}$ & $2,88 \mathrm{a}$ & $2,93 \mathrm{a}$ & $2,96 \mathrm{a}$ \\
\hline SST & $8,17 \mathrm{a}$ & $7,83 \mathrm{a}$ & $8,35 \mathrm{a}$ & $9,10 \mathrm{a}$ & $7,40 \mathrm{a}$ & $8,30 \mathrm{a}$ & $10,10 \mathrm{a}$ & $9,75 \mathrm{a}$ & $8,95 \mathrm{a}$ & $8,25 \mathrm{a}$ \\
\hline ATT (\%) & $1,38 \mathrm{a}$ & $1,40 \mathrm{a}$ & $1,36 \mathrm{a}$ & $1,57 \mathrm{a}$ & $1,25 \mathrm{a}$ & $1,51 \mathrm{a}$ & $1,58 \mathrm{a}$ & $1,41 \mathrm{a}$ & $1,42 \mathrm{a}$ & $1,51 \mathrm{a}$ \\
\hline
\end{tabular}

*Promedios con letras distintas son significativamente diferentes, según la prueba de Tukey $(P \leq 0,05)$. 
sacarosa, la pérdida de peso y la intensidad respiratoria, indicando que la acidez disminuye durante el desarrollo del fruto porque en la respiración se degradan ácidos orgánicos (Kays, 2004). Estos resultados concuerdan con el análisis de componentes principales realizado en frutos de feijoa por Gallego et al. (2003), en el que se determinó que la ATT es una variable importante en los cambios durante la maduración.

La falta de diferencia entre los tratamientos aplicados sobre la calidad del fruto comprueba que los factores que más controlan la calidad del fruto son fijados genéticamente (Mengel et al., 2001).

\section{Frutos cuajados}

Para el inicio del ensayo se tuvo en cuenta el número de flores inducidas del estudio anterior (García y Dueñez, 2007). Para el posterior análisis frente a los resultados obtenidos en el presente ensayo, fue importante analizar el alto porcentaje de frutos cuajados, debido a que la feijoa por ser una mirtácea, posee una capacidad de producción floral alta, la cual a su vez es regulada por la misma fisiología de la planta mediante abortos o pérdidas florales durante el periodo trascurrido desde su diferenciación floral al cuajado (García y Duéñez, 2007). Adicionalmente, existen pérdidas de flores y frutos a causa de variables climáticas (lluvia, veranos fuertes, humedad alta, etc.) y efectos mecánicos dentro del cultivo (labores culturales, aplicaciones, agentes polinizadores, etc.).

El análisis de varianza mostró diferencia significativa entre los tratamientos; el tratamiento con mejor resultado fue $\mathrm{KH}_{2} \mathrm{PO}_{4}$ al 0,5\%; continuando con la misma tendencia que se observó durante todo el ensayo en comparación con el testigo (tabla 3). El número de flores formadas con $\mathrm{KH}_{2} \mathrm{PO}_{4}$ al 0,5\% fue 197 mientras que con el testigo fue 44, y para frutos cuajados con $\mathrm{KH}_{2} \mathrm{PO}_{4}$ al $0,5 \%$ se obtuvo 136 mientras que con el testigo 28; por lo que la diferencia entre este producto y el testi- go tiene una relación de 4:1. Este resultado concuerda con lo reportado de otros cultivos en los cuales una aplicación de fósforo aumentó la floración, que además del incremento del metabolismo en estas yemas, el P fomenta la absorción de Mg que también es fundamental en la formación floral y, asimismo, promueve la síntesis de los ácidos nucleicos (Feucht, 1982). También, la respuesta favorable de la feijoa a la aplicación de $\mathrm{KH}_{2} \mathrm{PO}_{4}$ coincide con lo encontrado por Reddy y Majmudar (1985) en variedades de mango en las cuales un mayor porcentaje de inducción floral se observó en las laterales de brotes que habían florecido o fructificado en la temporada anterior y contenían las concentraciones más altas de fósforo en los brotes.

Los valores de frutos de feijoa cuajados (tabla 4) son mayores que los de frutales caducifolios drupáceas (duraznero, ciruelo europeo) en los cuales las variedades cuajan en un 25\% (Schumacher, 1985).

Los tratamientos respondieron de forma similar al número de flores formadas en el testigo; a pesar de que trascurrido el tiempo no hubo un aumento proporcional al número de flores formadas, sino que presentaron una tendencia estable a través del tiempo por lo que los productos aplicados no influyeron en el número de flores formadas (García et al., 2008).

Las aplicaciones con $\mathrm{KNO}_{3}$ mostraron efectos inferiores al tratamiento testigo (tabla 3). En contraste a estos resultados, Dennis (1996) y Faust (1989) confirman el aporte de nitrógeno como un elemento crucial en el cuajado de frutales caducifolios; en la feijoa, el $\mathrm{KNO}_{3}$ no actuó favorablemente por lo cual se supone que no existió una deficiencia en este nutriente durante el cuajamiento de los frutos. Por otro lado y según Agustí (2003) se deben evitar aplicaciones exageradas de fertilizantes, sobre todo si se utilizan nitratos, durante la fase del cuajado del fruto, ya que con ellas se puede inducir un desarrollo vegetativo excesivo que compite con la fructificación. 
Tabla 3. Efecto del nitrato de potasio $\left(\mathrm{KNO}_{3}\right)$, fosfato de potasio $\left(\mathrm{KH}_{2} \mathrm{PO}_{4}\right)$ y ethephon (Ethrel) en el número de flores inducidas, número de frutos cuajados y porcentaje de frutos cuajados de feijoa, 'Clon 41' (Quimba), promedio de tres ramas por árbol.

\begin{tabular}{|c|c|c|c|}
\hline \multirow{2}{*}{ Tratamiento } & \multirow{2}{*}{$\begin{array}{l}\text { Flores formadas } \\
\qquad(\text { No. })^{*}\end{array}$} & \multicolumn{2}{|c|}{ Frutos cuajados } \\
\hline & & (No.) & $(\%)$ \\
\hline $\mathrm{KNO}_{3} 1,0 \%$ & $30 a b$ & $15 d^{* *}$ & 50 \\
\hline $\mathrm{KNO}_{3} 1,5 \%$ & $50 a b$ & $20 \mathrm{~cd}$ & 40 \\
\hline $\mathrm{KNO}_{3} 2,0 \%$ & $26 b$ & $22 \mathrm{~cd}$ & 85 \\
\hline $\mathrm{KH}_{2} \mathrm{PO}_{4} 0,5 \%$ & 197 a & $136 \mathrm{a}$ & 69 \\
\hline $\mathrm{KH}_{2} \mathrm{PO}_{4}, 0,75 \%$ & $41 \mathrm{~b}$ & $35 \mathrm{abc}$ & 85 \\
\hline $\mathrm{KH}_{2} \mathrm{PO}_{4} 1,0 \%$ & $16 \mathrm{~b}$ & $10 d$ & 63 \\
\hline Ethephon $100 \mathrm{mg} \cdot \mathrm{L}^{-1}$ & $138 a b$ & $64 a b$ & 46 \\
\hline Ethephon $150 \mathrm{mg} \cdot \mathrm{L}^{1}$ & $66 a b$ & $21 \mathrm{~cd}$ & 32 \\
\hline Ethephon $200 \mathrm{mg} \cdot \mathrm{L}^{-1}$ & $18 \mathrm{~b}$ & $12 d$ & 67 \\
\hline Testigo & $44 a b$ & $28 a b c$ & 64 \\
\hline
\end{tabular}

*Datos obtenidos por García y Dueñez (2007)

* *Promedios con letras distintas son significativamente diferentes, según la prueba de Tukey $(P \leq 0,05)$.

\section{Frutos cosechados}

Al finalizar el ensayo se realizó un conteo de los frutos cosechados y se compararon con frutos los frutos cuajados (tabla 3). Para analizar el porcentaje de abortos florales y pérdida por otras variables (clima, nutrición, agua, etc.) se tuvo en cuenta los promedios de las repeticiones de cada ensayo (tabla 4). Se observó que el tratamiento que mayor número de frutos cosechados (73) presentó, fue el $\mathrm{KH}_{2} \mathrm{PO}_{4}$ al 0,5\%, el cual, a pesar de haber tenido una pérdida de frutos cercana a $54 \%$, mantuvo un buen desarrollo; similar caso ocurrió con el ethephon a $100 \mathrm{mg} \cdot \mathrm{L}^{-1}$, el cual, a pesar de haber tenido una pérdida floral muy alta, mantuvo una buena producción de frutos al final del ensayo.

El tratamiento $\mathrm{KH}_{2} \mathrm{PO}_{4}$ al $0,5 \%$ indujo una alta cantidad de botones florales (tabla 3), la cual mantuvo en una alta proporción durante el cuajado del fruto y manteniendo esta tendencia en la cosecha (tabla 4). Es evidente que se produjo un porcentaje de pérdida de fruto cuajado a fruto cosechado alto, del $56 \%$ en este tratamiento, lo cual, en general, justifican García y Dueñez (2007) como debido a factores fisiológicos, nutricionales, ambientales o mecánicos que no hicieron parte de este estudio. Los tratamientos restantes se comportaron de una forma menos eficiente, siendo ethephon a $100 \mathrm{mg} \cdot \mathrm{L}^{-1} \mathrm{el}$ segundo mejor tratamiento con 41 frutos cosechados, y todavía marcadamente mayor que el testigo (12), por lo que la diferencia entre este tratamiento $y$ el testigo tiene una relación 3:1.

Estos valores se asemejan a lo reportado por Agustí (2003) en duraznero, en donde 30 a 35\% de las flores formadas acaba siendo un fruto maduro. En manzano este porcentaje desciende hasta el 5\%, mientras en algunas naranjas se han registrado porcentajes inferiores al $1 \%$ de las flores formadas. Además, de los resultados se puede deducir que los tratamientos sin fósforo adicional no fueron capaces de nutrir al máximo los frutos en desarrollo (Agustí, 2003), sin embargo, exceso de este elemento $(0,75$ o $1,0 \%)$ igualmente causaría un menor número de frutos cosechados. 
Tabla 4. Efecto del nitrato de potasio $\left(\mathrm{KNO}_{3}\right)$, fosfato de potasio $\left(\mathrm{KH}_{2} \mathrm{PO}_{4}\right)$ y ethephon (Ethrel) en el número de frutos cuajados y cosechados con sus respectivos porcentajes en feijoa, 'Clon 41' (Quimba), promedio de tres ramas por árbol.

\begin{tabular}{|c|c|c|c|c|c|}
\hline Tratamiento & $\begin{array}{l}\text { Frutos } \\
\text { cuajados } \\
\text { (No.) }\end{array}$ & $\begin{array}{l}\text { Frutos } \\
\text { cosechados } \\
\text { (No.) }\end{array}$ & $\begin{array}{c}\text { Frutos cosechados } \\
\text { ( } \% \text { de frutos } \\
\text { cuajados) }\end{array}$ & $\begin{array}{c}\text { Frutos cosechados } \\
\text { ( } \% \text { de flores } \\
\text { formadas) }\end{array}$ & $\begin{array}{c}\text { Frutos caídos } \\
\qquad(\%)\end{array}$ \\
\hline $\mathrm{KNO}_{3} 1,0 \%$ & $15 d^{*}$ & $9 d$ & 60 & 30 & 40 \\
\hline $\mathrm{KNO}_{3} 1,5 \%$ & $20 \mathrm{~cd}$ & $11 \mathrm{~cd}$ & 55 & 22 & 45 \\
\hline $\mathrm{KNO}_{3} 2,0 \%$ & $22 \mathrm{~cd}$ & $9 d$ & 41 & 35 & 59 \\
\hline $\mathrm{KH}_{2} \mathrm{PO}_{4} 0,5 \%$ & $136 a$ & $73 a$ & 54 & 37 & 56 \\
\hline $\mathrm{KH}_{2} \mathrm{PO}_{4}, 0,75 \%$ & $35 a b c$ & $17 \mathrm{~cd}$ & 49 & 41 & 51 \\
\hline $\mathrm{KH}_{2} \mathrm{PO}_{4} 1,0 \%$ & $10 \mathrm{~d}$ & $5 d$ & 50 & 31 & 50 \\
\hline Ethephon $100 \mathrm{mg} \cdot \mathrm{L}^{-1}$ & $64 a b$ & $41 \mathrm{ab}$ & 64 & 34 & 36 \\
\hline Ethephon $150 \mathrm{mg} \cdot \mathrm{L}^{-1}$ & $21 \mathrm{~cd}$ & $16 \mathrm{~cd}$ & 76 & 41 & 24 \\
\hline Ethephon $200 \mathrm{mg} \cdot \mathrm{L}^{-1}$ & $12 d$ & $4 d$ & 33 & 22 & 67 \\
\hline Testigo & $28 a b c$ & $12 \mathrm{~cd}$ & 43 & 27 & 57 \\
\hline
\end{tabular}

*Promedios con letras distintas son significativamente diferentes, según la prueba de Tukey $(P \leq 0,05)$.

\section{CONCLUSIONES}

- Durante el ensayo se determinó que existe una respuesta favorable en diámetro longitudinal y transversal del fruto con respecto al tratamiento $\mathrm{KH}_{2} \mathrm{PO}_{4}$ al $0,5 \%$.

- Así mismo, 0,5\% $\mathrm{KH}_{2} \mathrm{PO}_{4}$ produjo el mayor número de frutos cuajados y cosechados.

- El ethephon, al $100 \mathrm{mg} \cdot \mathrm{L}^{-1}$ indujo la producción de los frutos de mayor peso fresco.
- Los productos aplicados no presentaron una alteración en las variables químicas ( $\mathrm{pH}$, sólidos solubles totales y acidez total titulable) de los frutos cosechados.

- La combinación del fosfato con el potasio $\left(\mathrm{KH}_{2} \mathrm{PO}_{4}\right)$ en baja concentración $(0,5 \%)$ fue muy favorable, mientras la de nitrato con el potasio $\left(\mathrm{KNO}_{3}\right.$ al 1,$\left.0 ; 1,5 \circ 2,0 \%\right)$ fue perjudicial para las variables estudiadas.

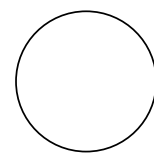

REFERENCIAS BIBLIOGRÁFICAS

Agustí, M. 2003. Citricultura. Mundi-Prensa, Madrid. 422 p.

Bhandal, I.S. y C.P. Malik 1988. Potassium estimation, uptake, and its role in the physiology and metabolism of flowering plants. Intl. Rev. Cytology 110 , 205-254.

Castelán-Estrada, M. y A.E. Becerril-Román. 1994. Fisiología de la producción forzada en Psidium guajava. Proc. Interamer. Soc. Trop. Hort. 38, 152-156.
Colorado, J. y S. Manjarrés. 1992. Evaluación del efecto de podas y fertilización con potasio en la inducción floral con pitahaya amarilla (Selenicereus megalanthus Schuman). Trabajo de grado. Facultad de Agronomía, Universidad Nacional de Colombia, Bogotá.

Corporación Colombia Internacional (CCI). 2000. La feijoa. Revista Exótica 4(4), 17-21.

Chacko, K. y G. Randhawa. 1974. Investigation on the use of (2-chloroethyl) phosphonic acid (ethephon, 
cepa) for the control of biennial bearing in mango. Scientia Hort. 2, 389-398.

Dennis, F.G, Jr. 1996. Fruit set. En: Kalb, K.M., P.K. Adrews, G.A. Lang y K. Mullinex (eds.). Tree fruit physiology: growth and development. Washington State Fruit Comission, Washington. pp. 99-106.

Faust, M. 1989. Physiology of temperate zone fruit trees. John Wiley \& Sons, Nueva York. 338 p.

Feucht, W. 1982. Das Obstgehölz. Eugen Ulmer, Stuttgart. $256 \mathrm{p}$.

Fischer, G. 2003. Ecofisiología, crecimiento y desarrollo de la feijoa. pp. 9-26. En: Fischer, G., D. Miranda, G. Cayón y M. Mazorra (eds). Cultivo, poscosecha y exportación de la feijoa (Acca sellowiana Berg). Produmedios, Bogotá. 152 p.

Galvis, J. 2003. Manejo de la cosecha y poscosecha de la feijoa. pp. 111-123. En: Fischer, G., D. Miranda, G. Cayón y M. Mazorra (eds.). Cultivo, poscosecha y exportación de la feijoa (Acca sellowiana Berg). Produmedios, Bogotá. 152 p.

Gallego, S.P., C.E. Riaño y L. Orozco. 2003. Determinación del comportamiento químico y fisiológico de Feijoa sellowiana en almacenamiento. Revista Cenicafe 54(1), 50-62.

García, O.J. y E.Y. Duéñez. 2007. Efecto del nitrato de potasio, fosfato de potasio y ethephon en la inducción floral y el cuajado del fruto de feijoa (Acca sellowiana [O.Berg] Burret). Trabajo de grado. Facultad de Agronomía, Universidad Nacional de Colombia, Bogotá.

García, O.J., E.Y. Duéñez, G. Fischer, B. Chávez y O.C. Quintero. 2008. El fosfato de potasio y el ethephon fomentan la inducción floral en feijoa o goiabeira serrana (Acca sellowiana [O. Berg] Burret). Revista Brasileira de Fruticultura 30 (en imprenta).

Grierson, W.- 2002. Fruit development, maturation, and ripening. En: Pessarakli, M. (ed.). Handbook of plant and crop physiology. Marcel Dekker, Nueva York. pp. 143-160.

IDEAM. 2002. Instituto de hidrológia, meteorología y estudios ambientales. Sistema de información ambiental, registros estaciónales sabaneta, Bogotá.

IGAC. 2000. Manual de códigos para levantamientos de suelos. Instituto Geográfico Agustín Codazzi, Bogotá.

Holdridge, L. 1996. Ecología basada en zonas de vida. Instituto Interamericano de Cooperación para la Agricultura (IICA). San José, Costa Rica.

Kays, S. 2004. Postharvest biology. Exon Press, Athens, Georgia. 568 p.

Lovatt, C.J. 1999. Management of foliar fertilization. Terra $17(3), 257-264$

Mengel, K., E.A. Kirkby, H. Kosegarten y T. Appel. 2001. Principles of plant nutrition. Kluwer Academic Publ., Dordrecht. 849 p.
Neilsen, G.H. y D. Neilsen. 2003. Nutritional requirements of apple. pp. 267-302. En: Ferree, D.C. y I.J. Warrington (eds.). Apples - Botany, production and uses. CABI Publishing, Wallingford, Oxon, U.K. 660 p.

Pachón, G. y O. Quintero. 1992. La feijoa (Feijoa sellowiana Berg) fruta promisoria para Colombia. Acta Hort. 310, 239-248.

Quintero, O. 2003. Selección de cultivares, manejo del cultivo y regulación de cosecha de feijoa. pp. 49-71. En: Fischer, G., D. Miranda, G. Cayón y M. Mazorra (eds.). Cultivo, poscosecha y exportación de la feijoa (Acca sellowiana Berg). Produmedios, Bogotá. 152 p.

Ramírez, O.P. 1997. Contribución a la selección de dos clones de feijoa (Acca sellowina Berg) mediante análisis sensorial y estudio en frigoconservación. Trabajo de grado. Facultad de Ingeniería, Universidad de la Sabana, Bogotá.

Reddy, S.E. y A.M. Majmudar. 1985. Tracking phosphorus patterns in mango (Mangifera indica L.) and possible relations to floral induction. Fertilizer Res. $6,225-234$

Rodríguez, M., H. Arjona y H. Campos. 2006a. Caracterización físico-química del crecimiento y desarrollo de los frutos de feijoa (Acca sellowiana Berg) en los clones 41 (Quimba) y 8-4. Agron. Colomb. 24(1), 56-61.

Rodríguez, M., H. Arjona y J.A. Galvis. 2006b. Maduración del fruto de feijoa (Acca sellowiana Berg) en los clones 41 (Quimba) y 8-4 a temperatura ambiente en condiciones de Bogotá. Agron. Colomb. 24(1) 68-76.

Salisbury, F.B. y C.W. Ross. 2003. Fisiología de las plantas: desarrollo de la plantas y fisiología ambiental. Vol. 3. Thompson Editores Spain, Paraninfo, Madrid. pp. 529-562.

Schumacher, R. 1989. Die Fruchtbarkeit der Obstgehölze. Verlag Eugen Ulmer, Stuttgart. 242 p.

Schumann, M. y P. Lüdders. 1992. Cultivation of feijoa: possibilities and limits in Colombia. Erwerbsobstbau 34(4), 110-112.

Seymor, G.B., J.E. Tucker y G.A. Tucker. 1993. Biochemistry of fruit ripening. Chapman $\&$ Hall, Londres. pp. 173-174.

Valderrama, J. K., G. Fischer y M.S. Serrano. 2005. Fisiología poscosecha en frutos de dos cultivares de feijoa (Acca sellowiana O. Berg Burret) sometidos a un tratamiento cuarentenario de frío. Agron. Colomb. 23(2), 276-282.

Weigel, D. 1995. The genetics of flower development: from floral induction to ovule morphogenesis. Annu. Rev. Genet. 29, 19-39.

Yahía, E.M., J.J. Ornelas y R. Ariza. 2006. El mango. Ed. Trillas, México. 224 p. 